\title{
Antibiotic Profile of Pathogenic Bacteria Isolated from Postsurgical Site Infections in Public Hospitals in Northern Jordan
}

\author{
Raed Ennab (1)', Waleed Al-Momani ${ }^{2}$, Rama Al-Titi ${ }^{2}$, Ayah Elayan ${ }^{2}$ \\ 'Department of Clinical Medical Sciences/General Surgery, Faculty of Medicine, Yarmouk University, Irbid, Jordan; ${ }^{2}$ Department of Basic Medical \\ Sciences/Microbiology, Faculty of Medicine, Yarmouk University, Irbid, Jordan \\ Correspondence: Raed Ennab, Department of Clinical Medical Sciences/General Surgery, Faculty of Medicine, Yarmouk University, Irbid, Jordan, \\ Tel +962797122559, Email Raed.ennab@yu.edu.jo
}

Background: Surgical site infections are hospital-acquired, vary from one hospital to another, and can cause significant postoperative morbidity, mortality, and prolonged hospital stay.

Objective: The main aim of the study was to identify the bacterial pathogens associated with surgical site infections and their antibiotic susceptibility in a public hospital in northern Jordan.

Methods: Postsurgical wound samples were collected and processed in a microbiology laboratory using standard microbiological techniques. Antibiotic susceptibility tests were performed using 13 antibiotics covering the gram-positive and gram-negative bacteria using the disc diffusion test.

Results: The bacterial species that were identified in this study include Escherichia coli 8 (29\%), Pseudomonas aeruginosa 3 (11\%), Proteus mirabilis 1 (3.5\%), Klebsiella pneumoniae 4 (14\%), Salmonella enterica 2 (7\%), Staphylococcus aureus 8 (29\%), Staphylococcus epidermidis 1 (3.5\%), and Streptococcus pyogenes 1 (4\%). The antibiotic profiles of these bacteria showed high resistance. The MAR indices showed that 17 of 28 bacteria isolated were above 0.2 indicating high resistance.

Conclusion: Resistant bacteria are becoming more dominant in wound infections with a high prevalence of multidrug resistant isolates. Hospital disinfection and treatment protocols regarding the use of antibiotics should be practiced vigorously and monitored regularly to combat resistance.

Keywords: surgical site infection, antimicrobial susceptibility, bacterial resistance, multidrug resistance, bacterial isolation, wound swab

\section{Background}

Surgical site infections (SSIs) are the main cause of postoperative morbidity and mortality. The Centres for Disease Control and Prevention (CDC) defines SSIs as infections that occur within a month of an operation in the part of the body where the surgery is done, or within 12 months if an implant is used. ${ }^{1,2}$ Among surgical patients, SSIs are the most common hospital-acquired infections, accounting for $36 \%$ of nosocomial infections. SSIs are associated with significant morbidity, mortality and increased costs in health care. ${ }^{3}$

Overall, SSIs are estimated to occur following $1-3.1 \%$ of all surgical procedures and are responsible for approximately $2.0 \%$ mortality due to health care-associated infections (HAIs). ${ }^{4}$ SSIs are often polymicrobial, including aerobic and anaerobic organisms. Staphylococcus aureus, Pseudomonas aeruginosa, the members of Enterobacteriaceae family, Streptococcus spp., Enterococcus spp., and Acinetobacter spp. are the predominant organisms that were reported. ${ }^{5}$

Risks factors associated with SSIs are either host related or perioperative factors: older patients, male sex, presence of comorbidities, smoking, using steroids, improper shaving technique of hair at the operative skin, as well as inadequate surgical scrub or antiseptic preparation have been shown to be associated with higher rates of SSIs. ${ }^{6,7}$ In addition, the 
higher bacterial load and the higher wound class (contaminated or dirty-infected cases) increase the probability of developing a wound infection..$^{8,9}$

However, a vast majority of these infections are preventable. In each case, a mechanical or anatomic source control maneuver is essential to the resolution of the surgical infection, and in most cases antimicrobial treatment is needed as well. Antibiotic prophylaxis administration prior to incision significantly reduces the incidence of SSIs. ${ }^{10}$

The presence of multidrug resistant strains, which is defined as an isolate with resistance to three or more antimicrobial classes, the polymicrobial nature of these infections, and the role of anaerobic bacteria in surgical site infections can cause failure in antibiotic therapy. ${ }^{5}$ Therefore, in order to achieve a high level of cure for these infections, causative microorganisms and their resistance to antimicrobial therapy must be identified.

At Jordan University Hospital 17\% of the patients had signs or symptoms of SSI within 30 days of surgery, of which $1.9 \%$ had SSI before being discharged from the hospital. ${ }^{11}$ In a retrospective study aimed to assess the frequency of SSIs, the bacterial profile, and the antibiogram of the isolates, from a tertiary hospital in Jordan found that SSIs constituting an overall incidence rate of about $5.4 \% .^{12}$

The current study aimed to detect and identify bacteria from surgical sites as well as to determine the antimicrobial susceptibility profile of these isolates among Jordanian patients from a public teaching hospital in northern Jordan.

\section{Materials and Methods}

\section{Study Population and Sample Size}

A hospital-based prospective study was carried out at Public-Teaching Hospital in northern Jordan with a total capacity of 400 beds. The study was carried out during the period from August 2019 to October 2019. The inclusion criterion was having clinical picture of a post-operative wound infection in different surgical wards and ICUs. The study included 24 patients with surgical site infections.

\section{Specimen Collection}

A surgical site infection was diagnosed by the attending physician before post-surgical wounds were swabbed during the 30 postoperative days under aseptic technique. The skin around the surgical wound was sterilized with $70 \%$ ethyl alcohol using a sterile cotton-wool swab. We avoided touching the surrounding tissues to prevent swab contamination with endogenous skin flora.

\section{Bacterial Isolation}

The swabs were transported to lab within an hour in Stuart's transport medium, then cultured on different culture media including blood agar, nutrient agar, MacConkey agar, and mannitol salt agar (Bio lab, Hungary), after which they were incubated aerobically for $24 \mathrm{hr}$ at $37^{\circ} \mathrm{C}$. The culture media were prepared and sterilized according to the manufacturer's instructions.

\section{Identification of Bacterial Isolates}

The Identification of the bacterial isolates depended upon the cultural, biochemical, and morphological characteristics according to Baron et al and Forbes et al. ${ }^{13,14}$ The biochemical identification tests differ according to the Gram reactivity; for Gram positive bacteria we performed coagulase and catalase tests, then checking the tolerance to novobiocin and the ability to grow on bile esculin agar. The bacitracin susceptibility test was used to differentiate Staphylococci from Micrococci. For Gram negative bacteria, the isolate was observed for morphology and motility and tested on triple sugar iron slant for the reaction pattern followed by tests using the triple sugar iron agar, methyl red, indole, citrate, and Voges Proskauer to confirm the identity of the isolate.

\section{Antibiotic Susceptibility Test}

Antibiotic susceptibility test was carried out using the disc diffusion method on Muller-Hinton agar according to the guidelines of the Clinical Laboratory Standards Institute "CLSI" using antibiotic disks including: penicillin $(10 \mu \mathrm{g})$, oxacillin $(1 \mu \mathrm{g})$, ampicillin $(10 \mu \mathrm{g})$, erythromycin $(15 \mu \mathrm{g})$, amoxicillin/clavulanic acid (20/10 $\mu \mathrm{g})$, ciprofloxacin $(5 \mu \mathrm{g})$, 
norfloxacin $(10 \mu \mathrm{g})$, cefepime $(30 \mu \mathrm{g})$, chloramphenicol $(30 \mu \mathrm{g})$, gentamicin $(10 \mu \mathrm{g})$, trimethoprim-sulfamethoxazole $(1.25 / 23.75 \mu \mathrm{g})$, tetracycline $(30 \mu \mathrm{g})$, clindamycin $(2 \mu \mathrm{g})$, and vancomycin $(30 \mu \mathrm{g})$. A brief suspension of the bacteria in saline was performed to a turbidity of $0.5 \mathrm{McFarland}$ standards. Then, the cell suspension was swabbed, and the swab was spread on the surface of a Mueller Hinton Agar plate (MHA) and left briefly to dry at room temperature. After that, the antibiotic disks were applied onto the agar; thereafter, they were incubated for $18-24$ hours at $35^{\circ} \mathrm{C}$. Lyophilized S. aureus (ATCC \# 25923) was used as a control. The interpretation of the results was in accordance with the guidelines of the Clinical and Laboratory Standards Institute. ${ }^{15}$

\section{Multiple Antibiotic Resistance}

Multiple antibiotic resistance (MAR) was calculated according to the following formula: Multiple antibiotic resistance index $=$ number of antibiotics to which organism is resistant to total number of antibiotics to which the organism is exposed. MAR index is indicative of the level of resistance of the isolated microorganisms.

\section{Results}

A total of 24 swab samples from surgical wounds with clinical suspicion of infection were collected and processed. Fifteen $(60 \%)$ were culture positive and $9(40 \%)$ were culture negative. Nine cases $(60 \%)$ with positive cultures showed the growth of a single organism, while $6(40 \%)$ had growth of multiple organisms. Twenty-eight bacterial species were isolated from $15(60 \%)$ of the 24 surgical wound samples. The isolated bacteria included: $6(21.4 \%)$ from bed sore patients who underwent minor surgeries before swapping, 9 (32\%) from diabetic foot patients who also underwent minor surgeries before swapping, 2 (7\%) isolates from post-operative orthopaedic surgeries, 6 (21.4\%) from tonsillectomy patients, $1(3.5 \%)$ isolate from gunshot patients, $3(11 \%)$ from trauma patients and $1(3.5 \%)$ from colectomy patients. The highest pathogenic bacteria isolation was detected in diabetic foot patients followed by bed sore and trauma patients, respectively. Distribution of the bacterial isolates with the type of surgery is presented in Table 1. Fifty-seven percent of the isolated bacteria were Gram negative, and $43 \%$ were Gram positive. Eight bacterial species were isolated and identified in this study, including: E. coli 8 (29\%), P. aeruginosa 3 (11\%), P. mirabilis 1 (3.5\%), K. pneumoniae 4 (14\%), S. enteric 2 (7\%) and S. aureus 8 (29\%), S. epidermidis 1 (3.5\%), S. pyogenes $1(4 \%)$.

The antibiotic profile of the isolates showed a high resistance as shown in Table 2. Only five isolates were sensitive to the 11 antibiotics used, while the other isolates were resistant to the same antibiotics with the range of 3-11 antibiotics. S. aureus was resistant to most of the antibiotics used. We found that $75 \%$ and $87 \%$ of $S$. aureus isolates were resistant to penicillin and

Table I Distribution of Bacterial Isolates with the Type of Surgery from Surgical Site Infections in Public Hospitals in Northern Jordan

\begin{tabular}{|c|c|c|c|c|c|c|c|c|c|c|c|c|c|c|c|}
\hline \multirow[t]{2}{*}{ Bacteria } & \multicolumn{2}{|c|}{ Bed Sore } & \multicolumn{2}{|c|}{ Diabetic Foot } & \multicolumn{2}{|c|}{ Fracture } & \multicolumn{2}{|c|}{ Tonsils } & \multicolumn{2}{|c|}{ Gun Shot } & \multicolumn{2}{|c|}{ Trauma } & \multicolumn{2}{|c|}{ Colectomy } & \multirow[t]{2}{*}{ No. of Isolates } \\
\hline & $\mathbf{M}$ & $\mathbf{F}$ & $\mathbf{M}$ & $\mathbf{F}$ & $\mathbf{M}$ & $\mathbf{F}$ & $\mathbf{M}$ & $\mathbf{F}$ & $\mathbf{M}$ & $\mathbf{F}$ & $M$ & $\mathbf{F}$ & $\mathbf{M}$ & $\mathbf{F}$ & \\
\hline E. coli & 2 & 0 & I & 2 & 0 & 0 & 3 & 0 & 0 & 0 & 0 & 0 & 0 & 0 & 8 \\
\hline P. mirabilis & 0 & 0 & I & 0 & 0 & 0 & 0 & 0 & 0 & 0 & 0 & 0 & 0 & 0 & 1 \\
\hline P. aeruginosa & 2 & 0 & 0 & 0 & 0 & 0 & 0 & 0 & I & 0 & 0 & 0 & 0 & 0 & 3 \\
\hline K. pneumonia & I & 0 & 0 & 1 & 0 & 0 & 2 & 0 & 0 & 0 & 0 & 0 & 0 & 0 & 4 \\
\hline S. enterica & 0 & 0 & I & 0 & 0 & I & 0 & 0 & 0 & 0 & 0 & 0 & 0 & 0 & 2 \\
\hline S. pyogenes & I & 0 & 0 & 0 & 0 & 0 & 0 & 0 & 0 & 0 & 0 & 0 & 0 & 0 & I \\
\hline S. aureus & 0 & 0 & 2 & I & I & 0 & 1 & 0 & 0 & 0 & I & I & I & 0 & 8 \\
\hline S. epidermidis & 0 & 0 & 0 & 0 & 0 & 0 & 0 & 0 & 0 & 0 & I & 0 & 0 & 0 & I \\
\hline Total & \multicolumn{2}{|c|}{6} & \multicolumn{2}{|c|}{9} & \multicolumn{2}{|c|}{2} & \multicolumn{2}{|c|}{6} & \multicolumn{2}{|c|}{ I } & \multicolumn{2}{|c|}{3} & \multicolumn{2}{|c|}{ I } & 28 \\
\hline
\end{tabular}

Abbreviations: M, male; F, female. 
Table 2 Antibiotic Profile of the Isolated Bacteria from Surgical Site Infections in Public Hospitals in Northern Jordan

\begin{tabular}{|c|c|c|c|c|c|c|c|c|c|c|c|c|c|c|c|c|c|c|c|c|c|c|c|c|}
\hline \multirow[t]{2}{*}{ Antibiotic } & \multicolumn{3}{|c|}{ S. enterica } & \multicolumn{3}{|c|}{ E. coli } & \multicolumn{3}{|c|}{ P. mirabilis } & \multicolumn{3}{|c|}{ P. aeruginosa } & \multicolumn{3}{|c|}{ K. pneumoniae } & \multicolumn{3}{|c|}{ S. pyogenes } & \multicolumn{3}{|c|}{ S. aureus } & \multicolumn{3}{|c|}{ S. epidermidis } \\
\hline & $\mathbf{S}$ & $\mathbf{R}$ & I & $\mathbf{S}$ & $\mathbf{R}$ & $\mathbf{I}$ & $\mathbf{S}$ & $\mathbf{R}$ & $\mathbf{I}$ & $\mathbf{S}$ & $\mathbf{R}$ & $\mathbf{I}$ & $\mathbf{S}$ & $\mathbf{R}$ & $\mathbf{I}$ & $S$ & $\mathbf{R}$ & I & $\mathbf{S}$ & $\mathbf{R}$ & I & $\mathbf{S}$ & $\mathbf{R}$ & I \\
\hline CIP & 2 & 0 & 0 & 5 & 2 & I & I & 0 & 0 & 3 & 0 & 0 & 4 & 0 & 0 & I & 0 & 0 & 5 & 3 & 0 & I & 0 & 0 \\
\hline AML & 1 & I & 0 & 4 & 4 & 0 & 0 & I & 0 & 0 & 3 & 0 & I & 3 & 0 & 1 & 0 & 0 & I & 7 & 0 & I & 0 & 0 \\
\hline NOR & 2 & 0 & 0 & 5 & 2 & I & I & 0 & 0 & 2 & I & 0 & 4 & 0 & 0 & 1 & 0 & 0 & 5 & 3 & 0 & I & 0 & 0 \\
\hline $\mathbf{E}$ & $\mathrm{N}$ & $\mathrm{N}$ & $\mathrm{N}$ & $\mathrm{N}$ & $\mathrm{N}$ & $N$ & $\mathrm{~N}$ & $\mathrm{~N}$ & $N$ & $N$ & $N$ & $\mathrm{~N}$ & $\mathrm{~N}$ & $\mathrm{~N}$ & $N$ & 0 & I & 0 & 4 & 4 & 0 & I & 0 & 0 \\
\hline C & 2 & 0 & 0 & 6 & 2 & 0 & I & 0 & 0 & 2 & I & 0 & 3 & I & 0 & 1 & 0 & 0 & 8 & 0 & 0 & I & 0 & 0 \\
\hline $\mathbf{C N}$ & 2 & 0 & 0 & 7 & I & 0 & I & 0 & 0 & 3 & 0 & 0 & 3 & I & 0 & I & 0 & 0 & 5 & 3 & 0 & I & 0 & 0 \\
\hline TE & 2 & 0 & 0 & 4 & 4 & 0 & 0 & I & 0 & 0 & 3 & 0 & 4 & 0 & 0 & 0 & I & 0 & 5 & 3 & 0 & I & 0 & 0 \\
\hline VA & $\mathrm{N}$ & $\mathrm{N}$ & $N$ & $\mathrm{~N}$ & $\mathrm{~N}$ & $\mathrm{~N}$ & $\mathrm{~N}$ & $\mathrm{~N}$ & $N$ & $\mathrm{~N}$ & $N$ & $\mathrm{~N}$ & $\mathrm{~N}$ & $\mathrm{~N}$ & $\mathrm{~N}$ & I & 0 & 0 & 7 & I & 0 & I & 0 & 0 \\
\hline AMC & 2 & 0 & 0 & 4 & 4 & 0 & I & 0 & 0 & 0 & 3 & 0 & 3 & 0 & I & I & 0 & 0 & 4 & 4 & 0 & 0 & I & 0 \\
\hline FEP & 2 & 0 & 0 & 5 & 3 & 0 & I & 0 & 0 & 3 & 0 & 0 & 3 & I & 0 & I & 0 & 0 & 5 & 3 & 0 & I & 0 & 0 \\
\hline $\mathbf{P}$ & 0 & 2 & 0 & 2 & 6 & 0 & 0 & I & 0 & 0 & 3 & 0 & 4 & 0 & 0 & I & 0 & 0 & 2 & 6 & 0 & 0 & I & 0 \\
\hline SXT & 2 & 0 & 0 & 4 & 4 & 0 & 1 & 0 & 0 & 0 & 3 & 0 & 3 & I & 0 & I & 0 & 0 & 5 & 3 & 0 & 1 & 0 & 0 \\
\hline OX & 0 & 2 & 0 & 6 & 2 & 0 & 0 & I & 0 & 0 & 3 & 0 & 0 & 4 & 0 & 0 & 1 & 0 & 4 & 4 & 0 & I & 0 & 0 \\
\hline
\end{tabular}

Abbreviations: S, sensitive; R, resistant; I, intermediate; N, not applicable; CIP, ciprofloxacin; AML, ampicillin; NOR, norfloxacin; E, erythromycin; C, chloramphenicol; CN, gentamicin; TE, tetracycline; VA, vancomycin; AMC, amoxicillin/clavulanic acid; FEP, cefepime; P, penicillin; DA, clindamycin; SXT, trimethoprim/sulfamethoxazole; OX, oxacillin.

ampicillin, respectively. Single S. pyogenes was resistant to tetracycline, erythromycin, and oxacillin, while S. epidermidis isolate was resistant to penicillin and ampicillin. One of the $P$. aeruginosa isolates was resistant to 8 of the antimicrobials, and the other two were resistant to 9 of the antimicrobials tested. $K$. pneumonia isolate showed a variable antimicrobial resistance, one isolate was resistant to 3 antimicrobials, 2 were resistant to 4 antimicrobials, and the last one was resistant to 9 tested antimicrobials. Most of $E$. coli isolates showed high antimicrobial resistance. Two isolates were resistant to 2 antimicrobials, one isolate was resistant to 7 antimicrobials, one isolate was resistant to 10 antimicrobials and 2 isolates were resistant to 11 of the 13 antimicrobials tested. A single P. mirabilis isolate was resistant to 6 of the tested antimicrobials. The most resistant bacteria to the studied antibiotics were E. coli, S. aureus, P. aeruginosa, K. pneumonia isolates which showed resistance to multiple antibiotics with a ratio of $0.92,0.85,0.75$ and 0.75 , respectively. The least resistant isolated bacteria were $S$. enteric which were resistant to only 2 of the tested antimicrobials namely penicillin and oxacillin. The most effective antibiotics against the isolated bacteria were ciprofloxacin, chloramphenicol, and gentamicin.

The multidrug resistance profile of the isolated bacteria to thirteen different antibiotics used in this study is shown in Table 3. The multiple antibiotic resistance (MAR) indices were determined with reference to the tested antibiotics, and it was above 0.2 in 17 of the total 28 bacteria studied. The highest MAR indices were detected in E. coli ( 0.92$)$, S. aureus (0.85) P. aeruginosa (0.75), K. pneumonia (0.75) and P. mirabilis (0.5). However, S. epidermides and S. pyogenes had MAR index less than 0.5 .

\section{Discussion}

Postoperative wound infection is a major complication in surgery, as it complicates the postoperative course, delays recovery, and results in prolonged hospital stay with subsequent increase in the cost especially with the presence of antimicrobial resistance. ${ }^{16}$ The aim of this study was to identify the various pathogens associated with surgical site infections and their antibiotic susceptibility in a public hospital in northern Jordan. The study included wound swabs 
Table 3 Multidrug Resistance Profile of the Isolated Bacteria to the Tested Antibiotics (N= 13)

\begin{tabular}{|l|c|c|l|c|c|}
\hline Parameter & Frequency & $\begin{array}{c}\text { MAR } \\
\text { Index }\end{array}$ & Parameter & Frequency \\
Index
\end{tabular}

Note: MAR index = multiple antibiotic resistance index = no. of antimicrobials to which the isolate is resistant/no. of antibiotics to which the isolate is subjected. Abbreviations: CIP, ciprofloxacin; AML, ampicillin; NOR, norfloxacin; E, erythromycin; C, chloramphenicol; CN, gentamicin; TE, tetracycline; VA, vancomycin; AMC, amoxicillin/clavulanic acid; FEP, cefepime; P, penicillin; DA, clindamycin; SXT, trimethoprim/sulfamethoxazole; OX, oxacillin.

collected from post-surgical wound infections. A total of 24 wound samples were collected and processed. Positive Cultures were higher than negative cultures, $15(60 \%)$ were culture positive and $9(40 \%)$ were culture negative showing the same type of bacterial species isolated by Al-Awaysheh et al ${ }^{12}$ who carried out a study to assess the bacterial profiles and the associated antibiotic susceptibility profiles in SSIs, which occurred in tertiary hospital in Amman, the capital of Jordan, SSIs constituted an overall incidence rate of about 5.4\%. ${ }^{12}$ Giacometti et al conducted a study on 676 surgery patients, pathogenic bacteria was identified in 614 individuals in which multiple etiologic agents were found in 343 patients, while a single agent was found in 271 patients. ${ }^{17}$ In a study carried out by Hubab et al, the growth percentages were $84 \%$ out of 250 wound swabs. ${ }^{18}$

In this study, the most commonly isolated pathogens were $S$. aureus 8 (29\%), E. coli 8 (29\%), K. pneumoniae 4 (14\%) and P. aeruginosa $3(11 \%)$. S. aureus is known to be predominant in surgical site infection across continents and over decades, and this is consistent with reports from other studies. It was isolated in $63 \%$ of patients in a study by Shriyan et al. ${ }^{19}$ In another study conducted by Manyahi, S. aureus was the predominant organism. ${ }^{20}$

In this study, 13 specimens (54\%) were from female patients and 11 (46\%) were from male patients. Among the 15 positive culture cases, $9(60 \%)$ belong to male patients and $6(40 \%)$ belong to female patients. Therefore, in this study wound infection was higher in male patients than in female patients, and this could be due to males being more active outdoors and exposed more to infectious agents. Anguzu and Olila in a study on 94 patients found that 56 (59.6\%) were males and $38(40.4 \%)$ were females. ${ }^{16}$ Similar results were shown in the study carried out by Khan et al where out of 104 patients, $64.4 \%$ were males and $35.6 \%$ were females. ${ }^{21}$ Also, in a study carried out by Adegoke et al there was a higher percentage of male $(76.5 \%)$ patients than females. ${ }^{22}$ The correct prescription for antibiotics depends upon antimicrobial susceptibility testing. The highest rate of resistance for ampicillin (70\%) within the Gram-positive isolates was in S. aureus, penicillin $(60 \%)$ and $40 \%$ for each of erythromycin, oxacillin and amoxicillin/clavulanic acid.

In this study, the predominant species were S. aureus and E. coli $(29 \%)$, which is in agreement with previous studies that reported S. aureus to be the most common cause of SSIs. ${ }^{23,24}$ S. aureus is part of the normal flora and can lead to 
endogenous infections. This is consistent with what has been reported previously by other studies. ${ }^{25}$ Endogenous fecal flora could be the reason for the observed high incidence of $E$. coli, in addition to poor hygiene. ${ }^{26}$ In a study on 63 university students in Jordan, nine bacterial species were isolated from the contact lenses, conjunctiva, and mobile phones. The isolates included gram positive and negative bacteria, and most of them were highly resistant to antibiotics. ${ }^{27}$ The rate of SSIs in the current study was comparable to that in other studies. However, the antibiogram was different than previously reported. ${ }^{28}$ This could be due to differences in the prevalence of bacterial strains among the population, variable infection-control measures and surgical techniques, and a high-likelihood of antibiotics misuse.

The multiple antibiotic resistance (MAR) indices were above 0.2 in 17 of the total 28 bacteria studied. This is comparable to other studies in the Middle East, where a retrospective study on nosocomial infections in Iran on 509 isolates showed that $80.5 \%$ and $57.3 \%$ in Gram-negatives and $91.7 \%$ and $72.9 \%$ in gram positives isolated strains were classified as multidrug-resistant (MDR) and extensive drug-resistant (XDR) strain, respectively. ${ }^{29}$

Limitations of this study include the convenient sample size and the lack of detection of anaerobes. Further studies on a larger sample size to investigate anaerobes in post-operative site infections are required. We used the STARD checklist when writing our report. ${ }^{30}$

\section{Conclusion}

Resistant bacteria are becoming more dominant in wound infections with a high prevalence of multidrug resistant isolates. Hospital disinfection and treatment protocols regarding the use of antibiotics should be practiced vigorously and monitored regularly to combat resistance.

\section{Data Sharing Statement}

We may share all the individual deidentified participant data upon request, keeping the privacy of the participants. The data may be shared by email or online in the form of a Microsoft Excel file containing the anonymized participant's ages, genders, clinical and microbiologic lab results. For data sharing please contact the corresponding author by email: raed. ennab@yu.edu.jo.

\section{Ethical Considerations}

- The study protocol was approved by the Institutional Ethics Committee at the Ministry of Health, Amman, Jordan. Approval number 2163/ 2019.

- Each participant gave a written informed consent.

- The study complies with the Declaration of Helsinki.

ClinicalTrials.gov Identifier: NCT05106803.

\section{Author Contributions}

All authors made a significant contribution to the work reported, whether that is in the conception, study design, execution, acquisition of data, analysis and interpretation, or in all these areas; took part in drafting, revising or critically reviewing the article; gave final approval of the version to be published; have agreed on the journal to which the article has been submitted; and agree to be accountable for all aspects of the work.

\section{Funding}

The authors received no financial support for the research, authorship, and/or publication of this article.

\section{Disclosure}

The authors declared no potential conflicts of interest with respect to the research, authorship, publication of this article, or this work. 


\section{References}

1. Sørensen LT, Hemmingsen U, Kallehave F, et al. Risk factors for tissue and wound complications in gastrointestinal surgery. Ann Surg. 2005;241:654-658. doi:10.1097/01.sla.0000157131.84130.12

2. Lenhardt R, Hopf HW, Marker E, et al. Perioperative collagen deposition in elderly and young men and women. Arch Surg. 2000;135:71-74. doi:10.1001/archsurg.135.1.71

3. Poggi JL. Perioperative strategies to prevent surgical-site infection. Clin Colon Rectal Surg. 2013;26:168-173. doi:10.1055/s-0033-1351133

4. Barie PS, Wilson SE. Impact of evolving epidemiology on treatments for complicated skin and skin structure infections: the surgical perspective. $J$ Am Coll Surg. 2015;220:105-116. doi:10.1016/j.jamcollsurg.2014.02.039

5. Akhi MT, Ghotaslou R, Beheshtirouy S, et al. Antibiotic susceptibility pattern of aerobic and anaerobic bacteria isolated from surgical site infection of hospitalized patients. Jundishapur J Microbiol. 2015;8:e20309. doi:10.5812/jjm.20309v2

6. Neumayer L, Hosokawa P, Itani K, et al. Multivariable predictors of postoperative surgical site infection after general and vascular surgery: results from the patient safety in surgery study. J Am Coll Surg. 2007;204:1178-1187. doi:10.1016/j.jamcollsurg.2007.03.022

7. Cruse PJ, Foord RA. Five-year prospective study of 23,649 surgical wounds. Arch Surg. 1973;107:206-210. doi:10.1001/ archsurg.1973.01350200078018

8. Segal CG, Waller DK, Tilley B, Piller L, Bilimoria K. An evaluation of differences in risk factors for individual types of surgical site infections after colon surgery. Surgery. 2014;156:1253-1260. doi:10.1016/j.surg.2014.05.010

9. Krizek TJ, Robson MC. Evolution of quantitative bacteriology in wound management. Am J Surg. 1975;130:579-584. doi:10.1016/0002-9610(75) 90516-4

10. Tanner J, Norrie P, Melen K. Preoperative hair removal to reduce surgical site infection. Cochrane Database Syst Rev. 2011;11:CD004122. doi:10.1002/14651858.CD004122.pub4

11. Bsisu I, Altibi A, Abusalem L, et al. Epidemiology of surgical site infections after gastrointestinal resection surgery in Jordan. The 3rd Jordanian Annual Surgical Clinical Congress. 2018.

12. Al-Awaysheh MM. Surgical site infections in a tertiary referral hospital in Amman: causative bacteria and antibiotic susceptibility. Jordan J Biol Sci Short Commun. 2018;11:231-233.

13. Baron EJO, Peterson LR, Finegold SM. Enterobacteriaceae. In: Bailey and Scotts-Diagnostic Microbiology. Missuri, USA: Mosby; $1994: 62-385$.

14. Forbes BA, Sahm DF, Weissfeld AS. Bailey and Scott's. In: St. Louis MO, editor. Diagnostic Microbiology. 12 ed. USA: Mosby Elsevier; 2007:778-781.

15. Clinical and Laboratory Standards Institute. Performance standards for antimicrobial susceptibility testing; twenty-sixth informational supplement. In: CLSI Document M100-S26. Wayne, PA: Clinical and Laboratory Standards Institute; 2016.

16. Anguzu JR, Olila D. Drug sensitivity patterns of bacterial isolates from septic post-operative wounds in a regional referral hospital in Uganda. Afr Health Sci. 2007;7:148-154. doi:10.5555/afhs.2007.7.3.148

17. Giacometti A, Cirioni O, Schimizzi AM. Epidemiology and microbiology of surgical wound infections. J Clin Microbiol. 2000;38:918-922. doi:10.1128/JCM.38.2.918-922.2000

18. Hubab M, Ullah O, Hayat A, et al. Antibiotic susceptibility profile of bacterial isolates from post-surgical wounds of patients in tertiary care hospitals of Peshawar, Pakistan. J Pak Med Assoc. 2018;68:1517-1521.

19. Shriyan A, Sheetal R, Nayak N. Aerobic micro-organisms in postoperative wound infections and their antimicrobial susceptibility patterns. $J$ Clin Diagn Res. 2010;4:3392-3399.

20. Manyahi J. Bacteriological Spectrum of Post-Operative Wound Infections and Their Antibiogram in a Tertiary Hospital. . Daressalaam, Tanzania: Dissertation Muhimbili University of Health and Allied Sciences; 2012.

21. Khan MS, Rehman S, Ali MA, Sultan B, Sultan S. Infection in orthopedic implant surgery, its risk factors and outcome. J Ayub Med Coll Abbottabad. 2008;20:23-25.

22. Adegoke A, Jacob S, Tom M, et al. Studies on multiple antibiotic resistant bacteria isolated from surgical site infection. Sci Res Essays. 2010;5:3876-3881.

23. Chakraborty SP, KarMahapatra S, Bal M, et al. Isolation and identification of vancomycin resistant Staphylococcus aureus from postoperative pus sample. Al Ameen J Med Sci. 2011;4:152-168.

24. Mulu W, Kibru G, Beyene G, et al. Postoperative nosocomial infections and antimicrobial resistance patterns of bacterial isolates among patients admitted at Felege Hiwot Referral Hospital, Bahirdar, Ethiopia. Ethiop J Health Sci. 2012;22:7-18.

25. Ahmed MI. Prevalence of nosocomial wound infection among postoperative patients and antibiotics patterns at teaching hospital in Sudan. $N \mathrm{Am}$ J Med Sci. 2012;4:29-34. doi:10.4103/1947-2714.92900

26. Malik S, Gupta A, Singh PK, et al. Antibiogram of aerobic bacterial isolates from post- operative wound infections at a tertiary care hospital in India. $J$ Infect Dis Antimicrob. 2011;28:45-51.

27. Al Momani W, Abu-Ismail L, Shihadah W, et al. Antimicrobial susceptibility of bacterial isolates from the conjunctiva, storage cases and mobile phones of university students using contact lenses. Contact Lens Anterior Eye. 2019;44:62-66. doi:10.1016/j.clae.2019.10.139

28. Vikrant NV, Pal SH, Juyal D, et al. Bacteriological profile of surgical site infections and their antibiogram: a study from resource constrained rural setting of Uttarakhand State, India. J Clin Diagn Res. 2015;9:DC17-DC20.

29. Sadeghi H, Khoei S, Bakht M, et al. A retrospective cross-sectional survey on nosocomial bacterial infections and their antimicrobial susceptibility patterns in hospitalized patients in northwest of Iran. BMC Res Notes. 2021;14:88. doi:10.1186/s13104-021-05503-0

30. Bossuyt PM, Reitsma JB, Bruns DE, et al. STARD 2015: an updated list of essential items for reporting diagnostic accuracy studies. BMJ. 2015;351:h5527. doi:10.1136/bmj.h5527 


\section{Publish your work in this journal}

Infection and Drug Resistance is an international, peer-reviewed open-access journal that focuses on the optimal treatment of infection (bacterial, fungal and viral) and the development and institution of preventive strategies to minimize the development and spread of resistance. The journal is specifically concerned with the epidemiology of antibiotic resistance and the mechanisms of resistance development and diffusion in both hospitals and the community. The manuscript management system is completely online and includes a very quick and fair peer-review system, which is all easy to use. Visit http://www.dovepress.com/testimonials.php to read real quotes from published authors.

Submit your manuscript here: https://www.dovepress.com/infection-and-drug-resistance-journal 